\title{
Letter to the Editor concerning "The effect of total hip arthroplasty on sagittal spinal-pelvic-leg alignment and low back pain in patients with severe hip osteoarthritis" by $W$. Weng et al. Eur Spine J (2016);25(11):3608-3614
}

\author{
Rajesh Kumar Rajnish $^{1} \cdot$ Prasoon Kumar $^{1} \cdot$ Sameer Aggarwal $^{1}$
}

Received: 17 April 2017/Accepted: 14 May 2017/Published online: 17 May 2017

(C) Springer-Verlag Berlin Heidelberg 2017

Dear Editor,

It was with great interest that we read the article entitled "The effect of total hip arthroplasty on sagittal spinalpelvic-leg alignment and low back pain in patients with severe hip osteoarthritis", published in November 2016 in the European Spine Journal [1]. The article was presented in the weekly journal club in our department at PGIMER, Chandigarh and we had an elaborate discussion regarding the same. We wish to commend the authors for bringing out this interesting facet of total hip arthroplasty.

Nevertheless, we have some pertinent points, which we would like to communicate to the authors. In this article the authors have dedicated entire focus on sagittal plane deformity as the sole cause of low back ache in patients of osteoarthritis (OA) hip. They did not consider the coronal plane deformity of spine and limb length discrepancy. A limb length deformity in OA hip can cause a compensatory coronal plane deformity and can lead to lateral bending of spine, which could be an additive to back pain. Total hip arthroplasty (THR) by correcting the limb length discrepancy improves the coronal plane abnormality, leading to decrease in functional spinal bend. This could be the cause of subsidence of pain. Hence, limb length discrepancy will influence the final outcome significantly [2].

The time interval between onset of back pain in OA patient and the THR has also not been commented upon by the authors. If the interval between symptoms and surgery is long, structural changes in the spine creep in and that could worsen the outcome. Such changes are often missed on a plane radiograph that the authors have used. A magnetic resonance imaging is often required for the same. So all these factors could have affected the final results in the study.

Moreover, preoperatively the authors have not quantified flexion deformity at hip joint by clinical examination, which could be a detrimental factor in outcome of low back pain [3].

\section{Compliance with ethical standards}

Conflict of interest The authors of this letter confirm that there are no conflicts of interest.

\section{References}

1. Weng W, Wu H, Wu M, Zhu Y, Qiu Y, Wang W (2016) The effect of total hip arthroplasty on sagittal spinal-pelvic-leg alignment and low back pain in patients with severe hip osteoarthritis. Eur Spine J 25(11):3608-3614

2. Yoshimoto H, Sato S, Masuda T, Kanno T, Shundo M, Hyakumachi T, Yanagibashi Y (2005) Spinopelvic alignment in patients with osteoarthrosis of the hip: a radiographic comparison to patients with low back pain. Spine (Phila Pa 1976) 30(14):1650-1657

3. Offierski CM, MacNab I (1983) Hip-spine syndrome. Spine (Phila $\mathrm{Pa}$ 1976) 8(3):316-321
Rajesh Kumar Rajnish

duktiraj@gmail.com

1 Postgraduate Institute of Medical Education \& Research, Chandigarh 160012, India 